\title{
MicroRNA suppression of stress-responsive NDRG2 during dexamethasone treatment in skeletal muscle cells
}

\author{
Bilal A. Mir, Rabia Islam, Ming Kalanon, Aaron P. Russell and Victoria C. Foletta* (D)
}

\begin{abstract}
Background: MicroRNAs (miRNAs) are increasingly being identified as modulatory molecules for physiological and pathological processes in muscle. Here, we investigated whether miRNAs influenced the expression of the stressresponsive gene $\mathrm{N}$-myc downstream-regulated gene 2 (Ndrg2) in skeletal muscle cells through the targeted degradation or translation inhibition of NDRG2 mRNA transcripts during basal or catabolic stress conditions.

Results: Three miRNAs, mmu-miR-23a-3p (miR-23a), mmu-miR-23b-3p (miR-23b) and mmu-miR-28-5p (miR-28), were identified using an in silico approach and confirmed to target the $3^{\prime}$ untranslated region of the mouse Ndrg 2 gene through luciferase reporter assays. However, miR-23a, -23b or -28 overexpression had no influence on NDRG2 mRNA or protein levels up to $48 \mathrm{~h}$ post treatment in mouse C2C12 myotubes under basal conditions. Interestingly, a compensatory decrease in the endogenous levels of the miRNAs in response to each other's overexpression was measured. Furthermore, dexamethasone, a catabolic stress agent that induces NDRG2 expression, decreased miR$23 \mathrm{a}$ and miR-23b endogenous levels at $24 \mathrm{~h}$ post treatment suggesting an interplay between these miRNAs and NDRG2 regulation under similar stress conditions. Accordingly, when overexpressed simultaneously, miR-23a, -23b and -28 attenuated the dexamethasone-induced increase of NDRG2 protein translation but did not affect Ndrg2 gene expression.
\end{abstract}

Conclusion: These findings highlight modulatory and co-regulatory roles for miR-23a, $-23 b$ and -28 and their novel regulation of NDRG2 during stress conditions in muscle.

Keywords: miRNA, NDRG2, Myotubes, Luciferase reporter assay, Stress response, Dexamethasone

\section{Background}

MicroRNAs (miRNAs) are small non-coding RNAs implicated as post-transcriptional regulators of fundamental skeletal muscle biological processes. Changes in their expression during muscle cell differentiation [1-3] and in response to stress and exercise [4-6] have resulted in the identification of miRNAs contributing to the control of muscle cell proliferation, tissue development, muscle regeneration and homeostasis [1, 3, 7-10]. MiRNAs may function through binding to specific regions of the $3^{\prime}$ untranslated region (3'UTR) of genes causing mRNA degradation or translational repression. For example, miR-23a binds to the 3'UTRs of fast myosin heavy isoforms

\footnotetext{
* Correspondence: victoria.foletta@deakin.edu.au

Institute for Physical Activity and Nutrition (IPAN), School of Exercise and Nutrition Science, Deakin University, Geelong, VIC 3222, Australia
}

contributing to the inhibition of myogenic differentiation [11]. MiR-23a also inhibits the translation of muscle-specific ubiquitin ligase genes, MAFbx/atrogin-1 and MuRF1, to protect against glucocorticoid dexamethasone-induced skeletal muscle atrophy [12]. Increased miR-181 expression enhances differentiation by suppressing homeobox protein HOX-A11, a MyoD negative regulator [3]. Other miRNAs and muscle-specific miRNAs (myomirs) are implicated in the control of skeletal muscle atrophy and hypertrophy processes [5, 13], myogenesis [14] and muscle-specific diseases (reviewed in [15]). While a number of miRNAs have been linked to the modulation of biological processes and stress responses in skeletal muscle, the genes they target to effect these changes are not well characterised.

$\mathrm{N}$-myc downstream-regulated gene 2 (NDRG2) is a stress-responsive member of the NDRG protein family 
involved in preventing tumour growth and invasion (reviewed in [16]). MiR-650 directly targets NDRG2 and its upregulation is inversely associated with decreased NDRG2 expression in colorectal [17] and breast cancer cells [18]. Hypoxia-induced miR-301a/b targets the 3'UTR of Ndrg2 resulting in NDRG2 protein suppression and increased autophagy and viability of prostate cancer cells $[19,20]$. MiR-181c overexpression binds Ndrg2's 3'UTR and downregulates its protein levels during cholangiocarcinogenesis and metastasis [21]. NDRG2 is also involved in a double-negative regulatory loop between leukemia inhibitory factor (LIF)/miR-181c where NDRG2 acts to inhibit LIF induction of miR-181c [21]. In adrenocortical carcinoma cells, miR-483-5p targets and suppresses NDRG2 to promote cancer invasion and pathogenesis [22]. Together, these studies highlight the interplay between miRNAs and NDRG2 function in cancer cells. There is currently very limited information regarding the regulation of NDRG2 by miRNAs in well-differentiated cell types such as skeletal muscle.

NDRG2 is well expressed in skeletal muscle [23] with expression increasing during muscle differentiation and development in vitro [24] and in vivo [25]. In muscle cells, NDRG2 promotes myoblast proliferation and protects against hydrogen peroxide-induced oxidative stress [26]. It is potentially associated with muscle mass changes where its expression is down and upregulated under anabolic and catabolic conditions, respectively, following dexamethasone treatment or resistance training [24]. The molecular factors regulating NDRG2 expression levels during myogenesis and in response to stress are poorly defined. While we identified the mouse Ndrg2 gene as a target of the peroxisome proliferator-activated receptor-gamma coactivator-1alpha and estrogen-related receptor alpha transcriptional program [27], a role for miRNA regulation of NDRG2 in skeletal muscle cells is currently unknown.

In this study, we used miRNA prediction software and literature analysis to identify possible miRNAs that target the $\mathrm{Ndrg} 2$ gene. Luciferase assays confirmed interactions of the predicted miRNAs with the mouse $N d r g 2$ 3'UTR. The modulation of endogenous mRNA and protein levels of NDRG2 under basal and dexamethasone stress conditions following individual or combined miRNA overexpression was investigated in $\mathrm{C} 2 \mathrm{C} 12$ myotubes.

\section{Materials and methods}

\section{MicroRNA target prediction using in silico approaches}

microRNA.org [28, 29] and miRWalk2.0 [30] softwares identified miRNAs predicted to target the 3'UTR region of mouse Ndrg2 (NM_013864). To note, all known mouse Ndrg2 variants have the same 3'UTR (https:// www.ncbi.nlm.nih.gov/gene/29811). microRNA.org uses
miRanda-predicted target sites with mirSVR scoring [28], and the miRWalk2.0 program enables the prediction of miRNA targets using a combination of the software programs: miRanda; miRWalk; RNA22; and Targetscan. MicroRNAs that were predicted both by microRNA.org and by all four software components of miRWalk2.0 were considered further. From these miRNAs, only those with a mirSVR score of -0.7 to -1.0 and an association with skeletal muscle biological processes in follow-up literature searches underwent further experimental validation.

\section{Dual luciferase reporter assay}

The full length $868 \mathrm{bp}$ 3'UTR fragment of the Ndrg2 mRNA containing predicted miRNA binding sites was amplified by RT-PCR. The 3'UTR product was cloned downstream of the NanoLuc luciferase (Nluc) gene coding region in the pmirNanoGLO2 vector, which also contains the firefly luciferase (luc2) reporter gene for normalisation (Promega, Alexandria, NSW, AUS). The oligonucleotides designed to encompass the Ndrg2 3'UTR seed sequences for the predicted miRNA binding sites and their mutated equivalents are listed in Table 1. Approximately $1 \times 10^{5}$ HEK293 cells (ATCC, Manassas, VA, USA) were plated in 96-well white-walled plates. The following day, $150 \mathrm{ng}$ of each plasmid and $5 \mathrm{nM}$ of each miRNA were co-transfected using Lipofectamine 2000 and Opti-MEM I reduced serum medium (Life Technologies, Mulgrave, VIC, AUS) as described by the manufacturer. Four hours post-transfection, the transfection mix was removed and replaced by growth medium containing $25 \mathrm{mM}$ glucose Dulbecco's Modified Eagle Medium (DMEM) with 10\% fetal bovine serum. Twenty-four or $48 \mathrm{~h}$ later, cells were consecutively assayed for Nanoluc and Firefly luciferase expression using the Nano-Glo ${ }^{\circ}$ Dual-luciferase ${ }^{\circ}$ Reporter assay kit (Promega) following the manufacturer's protocol. Normalized relative luciferase activity (RLA) was calculated as the following formula: RLA $=[N l u c] /[l u c 2$ luciferase $]$. To note, $\mathrm{C} 2 \mathrm{C} 12$ myoblasts were also assessed but HEK293 cells were consistently more reliable with their signal output and sensitivity than following transfection of the $\mathrm{C} 2 \mathrm{C} 12$ myoblasts.

\section{Cell culture and miRNA transfections}

Mouse C2C12 myoblasts (ATCC) were cultured in growth medium at $37^{\circ} \mathrm{C}$ in $5 \% \mathrm{CO}_{2}$. To differentiate cells, medium consisting of $2 \%$ horse serum and $25 \mathrm{mM}$ glucose DMEM was added when cells were confluent and refreshed every $48 \mathrm{~h}$ over a 6 day period. The miRNAs, mmu-miR-28-5p, mmu-miR-23a-3p, mmu-miR-23b-3p, mmu-miR-181a-5p or negative control (NC) miRNA (mirVana ${ }^{\circ}$ miRNA mimics; Thermo Fisher Scientific, Scoresby, VIC, AUS) were transfected at $5 \mathrm{nM}$ into 
Table 1 Oligonucleotide sequences used for cloning of mouse Ndrg2 3'UTR, the predicted miRNA seed sites or their scrambled mutated versions

\begin{tabular}{ll}
\hline Oligonucleotide: & Sequence $5^{\prime}-3^{\prime}:$ \\
\hline mNdrg2_3'UTR-F: & caccgctagcatgaccctcattgccttggtg \\
mNdrg2_3'UTR-R: & cgatctagaaacaatgctgttcagtttcctcta \\
miR23seed-F: & caccgctagcggtgggtcagtgatccttaatgtgatagaaatatccgcggg \\
miR23seed-R: & cgatctagaggatatttctatcacattaaggatcactgacccaccg \\
miR23mut-F: & caccgctagcggtgggtcagaatgttagtctcatgtagaaatatccgcggg \\
miR23mut-R: & cgatctagaggatatttctacatgagactaacattctgacccaccgagct \\
miR28seed-F: & caccgctagcttaacctgtgatatcctctagctcctaggtgaggccgcggg \\
miR28seed-R: & cgatctagaggcctcacctaggagctagaggatatcacaggttaagagct \\
miR28mut-F: & caccgctagcttaacctgttcatcgttactcactgcaggtgaggccgcggg \\
miR28mut-R: & cgatctagaggcctcacctgcagtgagtaacgatgaacaggttaagagct \\
\hline
\end{tabular}

differentiated myotubes using Lipofectamine 2000 and Opti-MEM I reduced serum medium (Life Technologies) as recommended. Myotubes were collected at 12, 24, or $48 \mathrm{~h}$ post transfection for NDRG2 mRNA and protein expression analyses. For mixed miRNA transfections, $5 \mathrm{nM}$ of the three miRNAs were each combined together and transfected into myotubes with comparison to $15 \mathrm{nM} \mathrm{NC}$ miRNA transfected samples. For catabolic treatments, $10 \mu \mathrm{M}$ dexamethasone (Sigma-Aldrich, Castle Hill, NSW, AUS) or $0.1 \%$ dimethyl sulfoxide (DMSO) vehicle were added to myotubes up to $48 \mathrm{~h}$ post-transfection and harvested concurrently.

\section{RNA extraction and gene expression analysis}

Total RNA was isolated using Tri-Reagent (Ambion Inc., Austin, TX, USA), treated with DNAse I (Life Technologies), and quantitated using the NanoDrop 1000 spectrophotometer (Thermo Fisher Scientific). Half a microgram of RNA was reverse-transcribed to form cDNA using the High Capacity cDNA reverse transcription kit (Applied Biosystems, Foster City, CA, USA) according to manufacturer's instructions. Semi-quantitative polymerase chain reaction (qPCR) was performed using the Mx3000 PCR system (Stratagene, La Jolla, CA, USA) with SYBR Green Master Mix (Applied Biosystems). The qPCR cycling conditions were: $95^{\circ} \mathrm{C}$ for $10 \mathrm{~min}(1 \mathrm{cycle}), 30 \mathrm{~s}$ at $95^{\circ} \mathrm{C}$ and $60^{\circ} \mathrm{C}$ for one min (40 cycles). Primers for mouse Ndrg2 (NM_013864.2; forward 5'cccacacagacctcgttcc and reverse 5 'gccatcgatggatgctgca) and a housekeeping gene, 36B4/Rplpo (NM_007475.5; forward 5'ttgtgggagcagacaatgtg and reverse 5'agtcctccttggtgaacacg), were synthesized by GeneWorks (Adelaide, SA, AUS). To note, the primers for $N d r g 2$ will detect all known variants of mouse Ndrg2. Samples were measured in duplicate using the Stratagene Mx3000P qPCR thermal cycler (Agilent Technologies, Mulgrave, VIC, AUS) and MxPRO qPCR software (Agilent Technologies). The relative gene expression is expressed as arbitrary units, which was calculated using the $2^{-\Delta C t}$ formula following normalization to $36 B 4$ cycle threshold $(\mathrm{Ct})$ levels.

\section{Western blotting}

Cells were lysed in $1 \times$ modified RIPA buffer $(50 \mathrm{mM}$ Tris- $\mathrm{HCl}, \mathrm{pH}$ 7.4, $150 \mathrm{mM} \mathrm{NaCl}, 0.25 \%$ deoxycholic acid, 1\% NP-40, 1 mM EDTA) (Merck Millipore, North Ryde, NSW, AUS) containing dilution of 1:1000 protease inhibitor cocktail (Sigma-Aldrich) and 1:100 HALT phosphatase inhibitor cocktail (Thermo Fisher Scientific). Protein lysate concentrations were determined using Pierce ${ }^{\mathrm{mi}}$ BCA Protein Assay Kit (Thermo Fisher Scientific). Ten or twenty micrograms of protein lysates were electrophoresed on either $10 \%$ SDS-PAGE gels and transferred to Immobilon ${ }^{\circ}$-FL polyvinylidene difluoride membrane (Merck Millipore, Kilsyth, VIC, AUS). Membranes were blocked in 5\% BSA for $1 \mathrm{~h}$ before incubation at $4{ }^{\circ} \mathrm{C}$ overnight in primary antibodies diluted in 5\% BSA/PBS. Rabbit polyclonal anti-NDRG2 antibodies (HPA002896; 1:5000) were obtained from Sigma-Aldrich. Rabbit monoclonal anti- $\alpha$-tubulin (clone DM1A; 1:5000) was obtained from Abcam (Cambridge, MA, USA). The detection of proteins was performed using goat anti-rabbit AlexaFluor ${ }^{\circ} 680$ or donkey anti-mouse AlexaFluor ${ }^{\circ} 800$ IgG antibodies (Life Technologies) diluted at 1:10,000 in 50\% Odyssey blocking buffer (Li-COR Biosciences, Lincoln, NE, USA), 50\% PBS and $0.01 \%$ SDS. Membranes were imaged using the infrared imaging system (Li-COR Biosciences). Proteins were normalized against $\alpha$-tubulin protein levels using the Li-COR software.

\section{Detection of miRNA expression}

MiRNA levels were measured by qPCR using specific primer and probes sets and the Taqman Universal MasterMix II, no UNG, kit as per the manufacturer's instructions (Applied Biosystems, Carlsbad, CA). For miRNA expression analyses, total RNA (50 ng) was 
reverse transcribed using the Taqman microRNA Reverse Transcription (RT) kit (Applied Biosystems). A customized RT primer pool was prepared by pooling all miRNA-specific stem-loop primers. In brief, miRNA-specific primers (TaqMan ${ }^{\bullet}$ MicroRNA assay kits, Life Technologies) were pooled and diluted in nuclease free water to obtain a final dilution of $0.05 \mathrm{x}$ each in a volume of $15 \mu \mathrm{l}$ containing $2 \mathrm{mM} \mathrm{dNTP}, 10 \mathrm{U}$ enzyme, $1 \times$ RT buffer, $4 \mathrm{U}$ RNase inhibitor and $50 \mathrm{ng}$ of total RNA. The reaction mix was reverse transcribed using the following conditions: $30 \mathrm{~min}$ at $16^{\circ} \mathrm{C}, 30 \mathrm{~min}$ at $42{ }^{\circ} \mathrm{C}$ and $5 \mathrm{~min}$ at $85^{\circ} \mathrm{C}$. The $\mathrm{qPCR}$ conditions consisted of 1 cycle of $10 \mathrm{~min}$ at $95^{\circ} \mathrm{C}$, and 40 cycles of $15 \mathrm{~s}$ at $95^{\circ} \mathrm{C}$ and $60 \mathrm{~s}$ at $60^{\circ} \mathrm{C}$. The TaqMan ${ }^{\mathrm{Tu}}$ microRNA snoRNA202 Control Assay was used as a normalizing control. Samples were run in triplicate for all miRNA targets and analysis was performed using the Stratagene MX3000P thermal cycler and dedicated software.

\section{Statistics}

All data are reported as the mean \pm standard error of the mean. Statistical differences were assessed using an unpaired Student's $t$ test for two-group comparisons at each time-point. For data involving three or more groups, data were subjected to one-way ANOVA with a Dunnett's or Sidak's multiple comparison test performed if a significant difference was found. Analyses were performed using GraphPad Prism (GraphPad Software version 7, La Jolla, CA, USA). Data were considered statistically significant if $p<0.05$.

\section{Results}

miR-23a, $-23 b$ and -28 bind and inhibit the translation of Ndrg2 3'UTR

Using an in silico approach, 16 miRNAs were initially predicted by both microRNA.org and miRWalk2.0. Following further consideration of their mirSVR score [28] and whether they had reported associations with skeletal muscle biological processes in the literature, three miRNAs; mmu-miR-23a-3p (miR-23a), mmu-miR-23b-3p (miR-23b), and mmu-miR-28-5p (miR-28), remained as predicted to bind to the murine Ndrg2 3'UTR with both miR-23a and -23b binding to the same seed site (Fig. 1a-b). To confirm whether these miRNAs targeted Ndrg2 3'UTR in vitro, luciferase assays revealed miR-23a, $-23 \mathrm{~b}$ and -28 overexpression decreased $N d r g 2$ 3'UTR-luciferase reporter activity by 30,35 and $44 \%$, respectively (Fig. 1c). To check the validity of our selection criteria, we also compared mmu-miR-181a-5p (miR-181a), which did not fit all the selection criteria. MiR-181a is associated with age-related muscle atrophy through binding to the Sirt1 gene [31], and was predicted to bind to Ndrg2 3'UTR in microRNA. org (mirSVR score of -1.0372). However, miR-181a was not identified using miRWalk2.0, and its overexpression had no impact on Ndrg2 3'UTR-luciferase reporter activity (Fig. 1c). To confirm the sites of interaction for the $3 \mathrm{miR}$ NAs within the mouse Ndrg2 3'UTR region, luciferase assays were repeated using sequences containing the predicted seed site domains for miR-23a/-23b and miR-28 binding. MiR-23a, -23b, and -28 overexpression each significantly reduced luciferase activity by 22,26 and $17 \%$, respectively, indicating an ability to bind to their respective seed sites. The reductions in luciferase activity were blocked when the miR-23a/-23b or miR-28 seed sequences were mutated to prevent miRNA binding (Fig. 1d-e).

\section{MiRNA overexpression effects on endogenous NDRG2 mRNA and protein levels}

MiR-23a, miR-23b and miR-28 were overexpressed in $\mathrm{C} 2 \mathrm{C} 12$ myotubes to evaluate if they could inhibit endogenous NDRG2 levels. Significant miRNA overexpression was achieved at each time point when compared to the respective negative control group (Additional file 1: Figure S1), which was included for each time point as it was noted that $\mathrm{Ndrg} 2$ gene expression changed transiently in response to transfection and/or differentiation effects. No change in Ndrg2 mRNA levels was measured at 12,24 or $48 \mathrm{~h}$ following miR-23a or -23b overexpression (Fig. 2a-b); however, Ndrg2 mRNA expression was reduced by $29 \%(p=0.018)$ at $24 \mathrm{~h}$ post miR-28 transfection (Fig. 2c). Furthermore, no decrease in NDRG2 protein levels at any time point following transfection of either of the three miRNAs was identified (Fig. 2d-f).

\section{Regulation of endogenous miR-23a, $-23 b$ and -28 levels following miRNA overexpression or dexamethasone treatment in C2C12 myotubes}

As the overexpression of miR-23a, $-23 b$ and -28 did not reduce NDRG2 protein levels, we next investigated whether miRNA overexpression could potentially compensate for each other reducing their ability to inhibit NDRG2 expression. MiRNA cooperativity and redundancy in biological processes is evident in C. elegans worm studies [32, 33] and in cardiac muscle tissue where multiple miRNAs may be required to target a common gene in order to regulate a biological process (reviewed in [34]). Unexpectedly, we observed that miR-23a overexpression in myotubes resulted in the significant downregulation of endogenous miR-23a and -28 levels at $12 \mathrm{~h}$ post transfection (Fig. 3a-b). Similarly, miR-23b overexpression reduced endogenous miR-23a levels significantly at $12 \mathrm{~h}$ (Fig. 3c). Furthermore, miR-28 overexpression decreased endogenous miR-23a and miR-23b levels at $12 \mathrm{~h}$ and $24 \mathrm{~h}$, respectively, post transfection (Fig. 3e-f).

A catabolic stress condition known to upregulate NDRG2 [24, 35] was next introduced. It has also been 
A

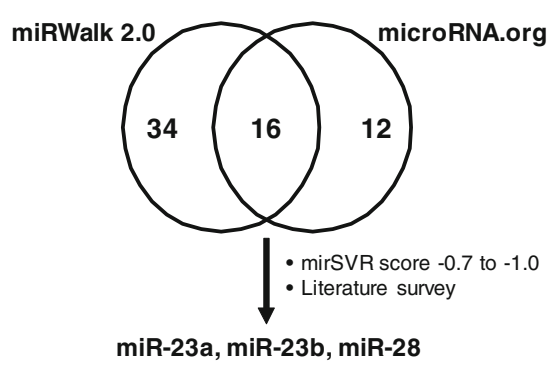

B

\begin{tabular}{|c|c|c|}
\hline 543 & 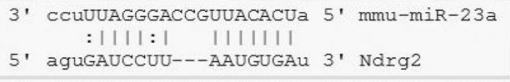 & mirsvR score: -0.7104 \\
\hline 543 & 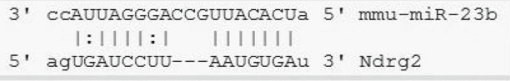 & mirSVR score: -0.7104 \\
\hline $164:$ & 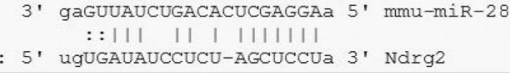 & mirsvR score: -0.9811 \\
\hline
\end{tabular}

C

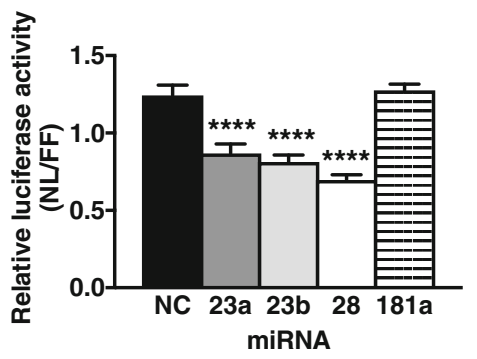

D

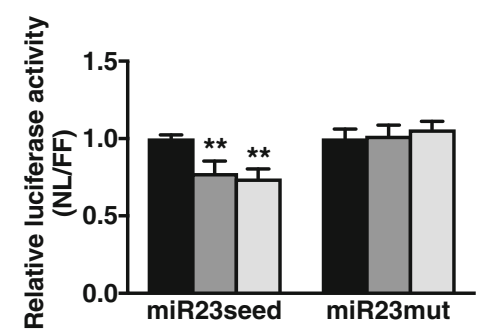

$\mathbf{E}$

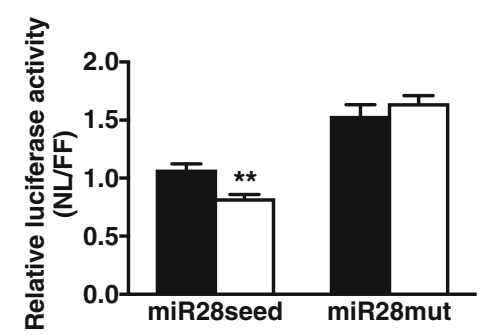

Fig. 1 Selection and validation of predicted miRNAs binding to the mouse Ndrg2 3'UTR. a Venn diagram of miRNAs predicted from miRWalk2.0 and microRNA.org algorithms, and selection criteria used to identify potential miRNAs binding Ndrg2 $3^{\prime} U T R$. b Nucleotide sequence alignment between the seed sites in the Ndrg2 $3^{\prime} U T R$ and predicted miRNAs with their corresponding mirSVR scores. c Dual-luciferase reporter assays determining the interaction of overexpressed miR-23a (dark grey bar), -23b (light grey bar), -28 (white bar), -181a (striped bar) and negative control (NC, black bar) with full-length Ndrg2 3'UTR; (d), with the miR-23a/-23b seed site (miR23seed) or its mutated version (miR23mut); and (e), with the miR-28 seed site (miR28seed) or its mutated version (miR28mut). Data is representative of three independent experiments with $n=5-6$ per sample group, and expression levels are presented as arbitrary units (AU). ${ }^{* *} p<0.01$ and ${ }^{* * *} p<0.0001$ to NC

reported that this catabolic stress, the glucocorticoid dexamethasone, decreases miR-23a levels in $\mathrm{C} 2 \mathrm{C} 12$ myotubes $48 \mathrm{~h}$ post treatment [36]. Here, we also observed a downregulation of miR-23a, but at $24 \mathrm{~h}$, not $48 \mathrm{~h}$ post dexamethasone treatment $(p<0.05$; Fig. 4a). In addition, miR-23b expression levels were decreased $24 \mathrm{~h}$ post treatment which was not sustained at $48 \mathrm{~h}(p<0.001$; Fig. $4 \mathrm{~b})$. No significant regulation by dexamethasone of miR-28
(Fig. 4c) or of the miRNA control gene, snoRNA202, was measured (Fig. 4d).

\section{Combined overexpression of miR-23a, $-23 b$ and -28 blocks NDRG2 induction during catabolic stress conditions}

As endogenous miR-23a, $-23 \mathrm{~b}$ and -28 levels were reduced by the overexpression of each other, and 

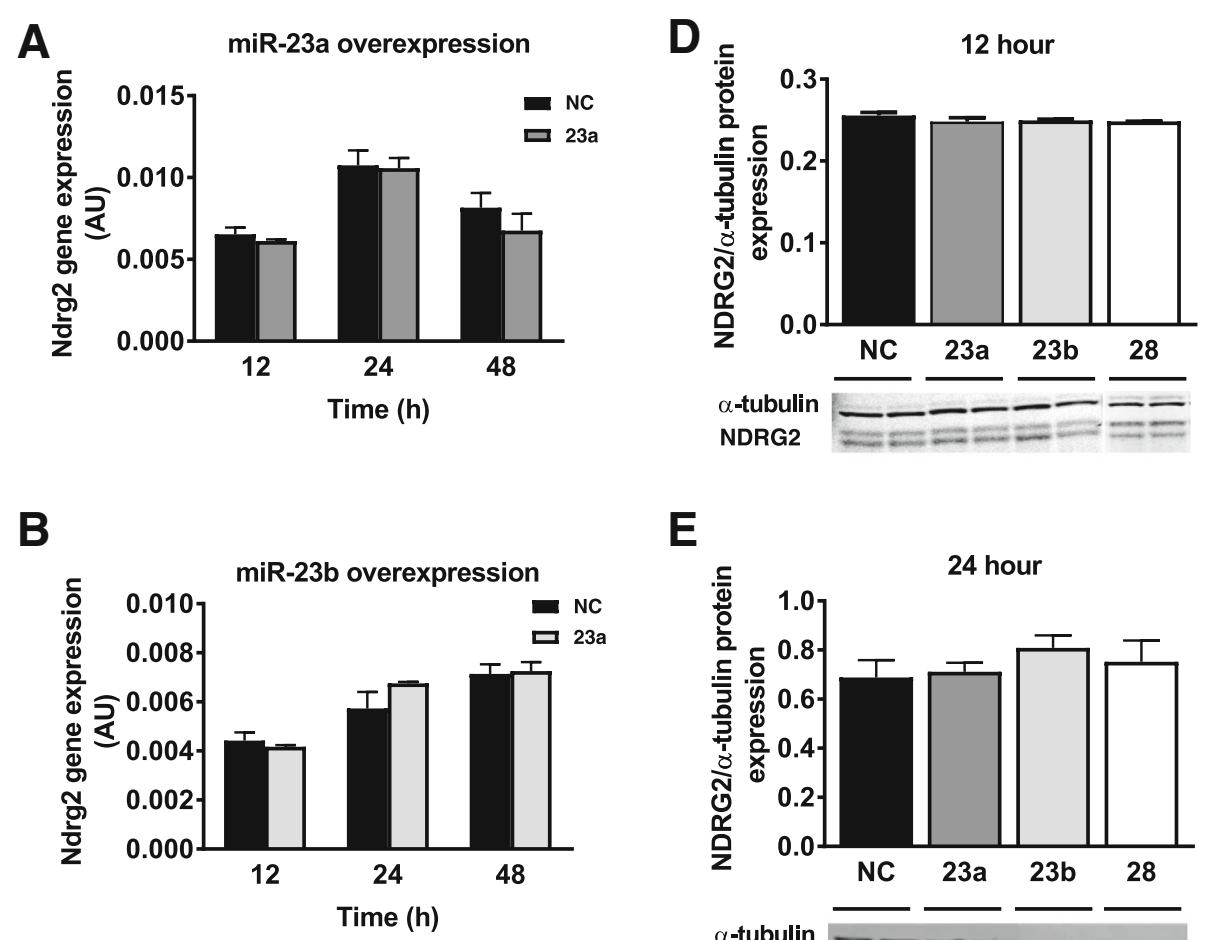

E
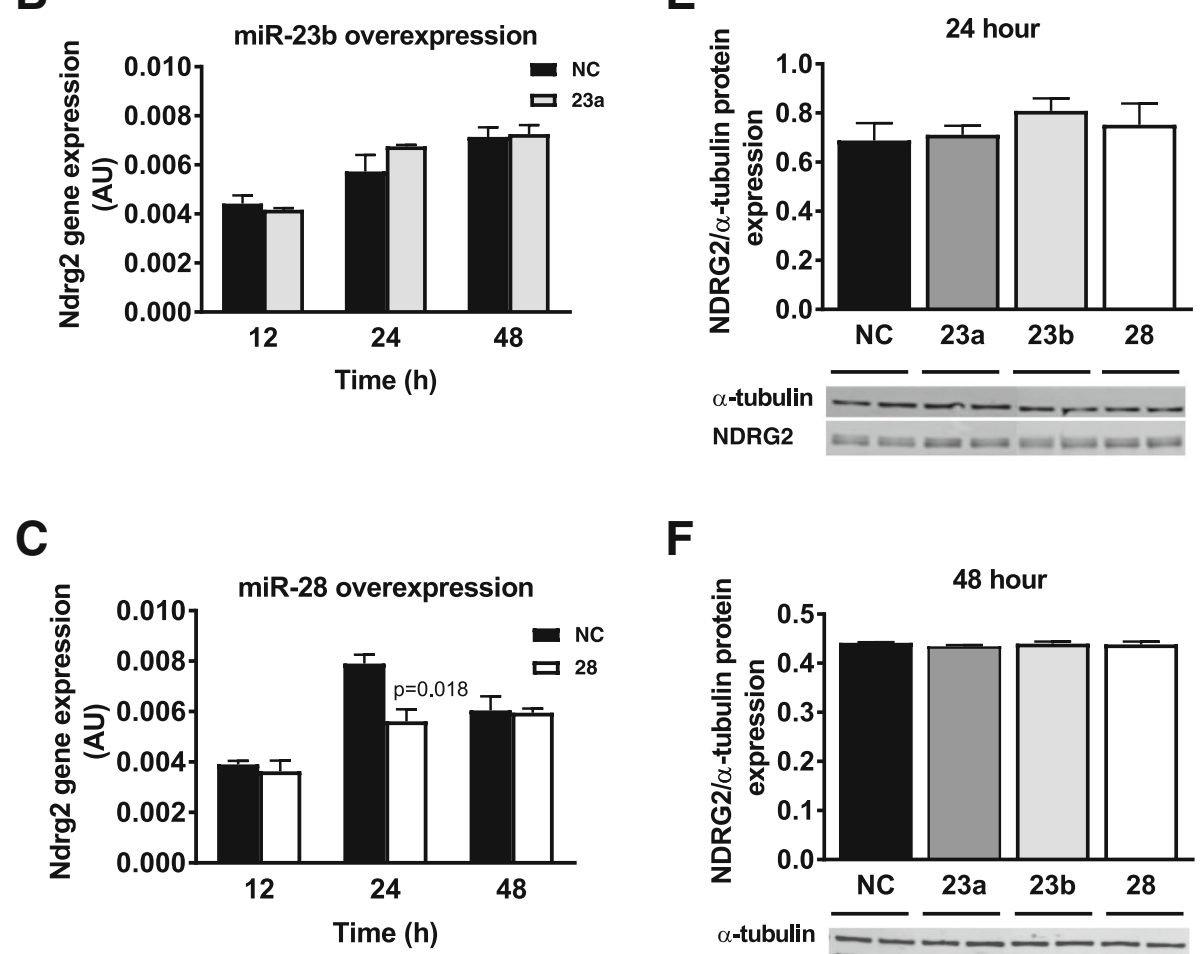

$\mathbf{F}$

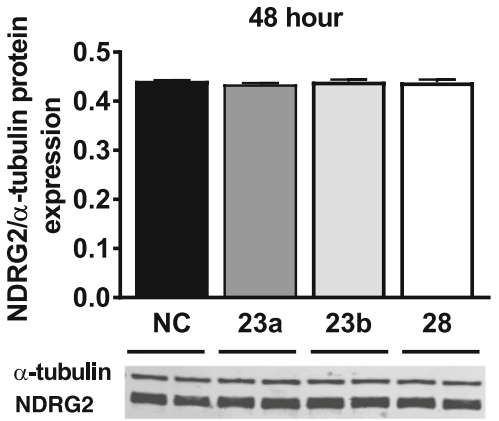

Fig. 2 Impact of miRNA overexpression on endogenous mRNA and protein levels of NDRG2 under basal conditions. Time-course in hours (h) of Ndrg2 mRNA expression following transfection of (a) miR-23a (dark grey bar), (b) miR-23b (light grey bar), and (c) miR-28 (white bar) compared to negative control (NC) miRNA (black bar). NDRG2 protein levels at (d) $12 \mathrm{~h}$, (e) $24 \mathrm{~h}$ and (f) $48 \mathrm{~h}$ post miRNA transfection. Data are representative of two to three independent experiments with $n=3-4$ per sample group, and expression levels are presented as arbitrary units (AU)

miR-23a and -23b decreased with dexamethasone treatment, these findings suggest an interplay between the three miRNAs and also with Ndrg2's stress-response regulation by dexamethasone [24, 35]. Therefore, a cocktail mix of the three miRNAs was transfected into myotubes to determine if their combined overexpression could regulate NDRG2 levels in the presence or absence of dexamethasone. Transfection of the miRNA cocktail mix achieved significant overexpression of miR-23a, $-23 \mathrm{~b}$ and -28 simultaneously in myotubes (Additional file 2: Figure S2); however, no change was observed in Ndrg2 mRNA or NDRG2 protein levels under basal conditions (Fig. 5a-b). In the presence of dexamethasone at $48 \mathrm{~h}$ post treatment, $N d r g 2$ mRNA and NDRG2 protein levels increased by approximately 100 and 20\%, respectively, in $\mathrm{C} 2 \mathrm{C} 12$ myotubes (Fig. 5c-d); however, the induction of NDRG2 protein by dexamethasone was blocked following the combined overexpression of the three miRNAs $(p=0.0012$; Fig. $5 \mathrm{~d})$. No impact on $N d r g 2$ mRNA expression under these same conditions was measured (Fig. 5c). 
A

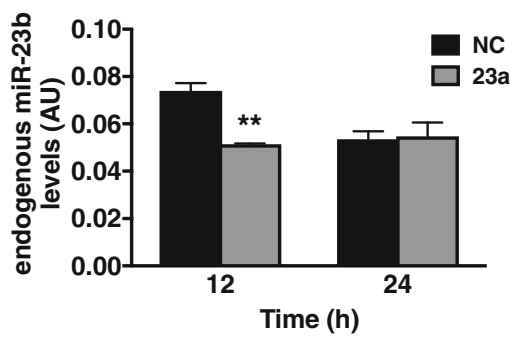

C

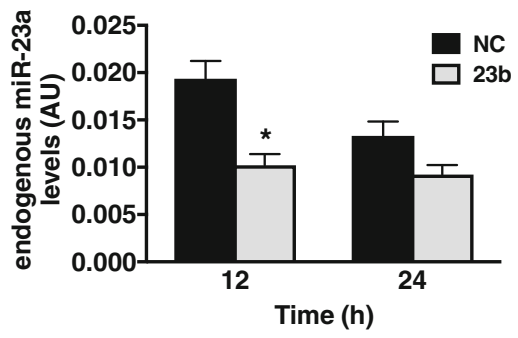

E

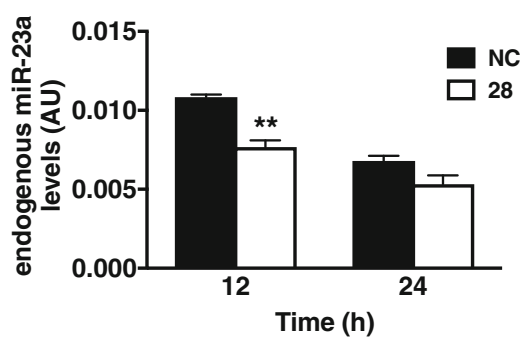

B

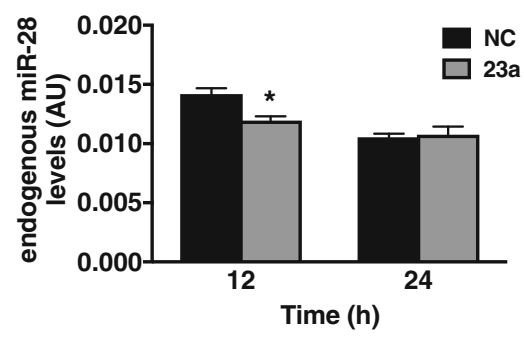

D

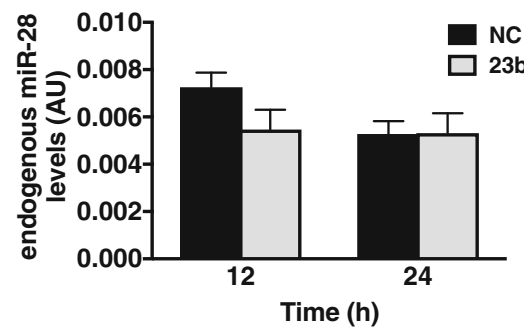

$\mathbf{F}$

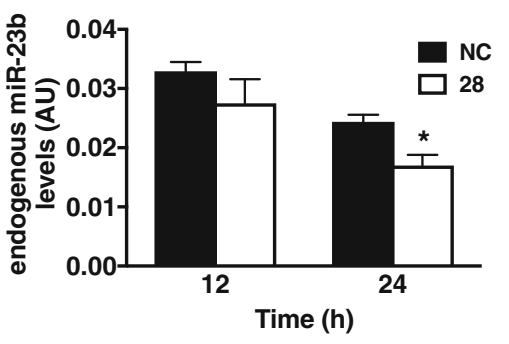

Fig. 3 MiRNA overexpression downregulates endogenous miR-23a, -23b and -28 levels. Endogenous miRNA levels over time (h) following miRNA overexpression. a Endogenous miR-23b and (b) miR-28 levels following miR-23a overexpression (dark grey bar). c Endogenous miR-23a and (d) miR-28 levels following miR-23b overexpression (light grey bar). e Endogenous miR-23a and (f) miR-23b levels following miR-28 overexpression (white bar). Data are representative of two independent experiments with $n=4$ per sample group; * $p<0.05$ and ${ }^{* *} p<0.01$ to negative control (NC; black bar) per respective time point, and expression levels are presented as arbitrary units (AU)

\section{Discussion}

The aim of this study was to identify a novel mechanism of NDRG2 regulation in skeletal muscle cells with a specific focus on miRNAs. Using an in silico approach, miR-23a, -23b and -28 were identified and their potential interaction with mouse $\mathrm{Ndrg} 23^{\prime} \mathrm{UTR}$ confirmed using cell-based biochemical assays. Individual or combined miRNA overexpression did not inhibit endogenous NDRG2 under basal conditions. However, the combined overexpression of the miRNAs inhibited the induction of NDRG2 protein expression following dexamethasone treatment. These results demonstrate a novel interplay between miRNAs and the regulation of NDRG2 under catabolic conditions in myotubes.

The importance of miRNAs in skeletal muscle development and homeostasis is highlighted in mice expressing a skeletal muscle-specific deletion of Dicer, an endonuclease required for miRNA processing. Mice unable to process miRNAs within skeletal muscle develop muscle hypoplasia, reduced muscle mass and perinatal death [37]. MiRNAs are also implicated in the control of muscle regeneration, dystrophies, myopathies, and in the development of cardiovascular and neurodegenerative diseases (reviewed in [15, 34]). How miRNAs function in muscle tissues is complex as they are reported to potentially 'buffer' against physiological and pathological changes to maintain homeostasis. To do this, they may target multiple gene transcripts in the same pathway to ensure a biological outcome, or display cooperativity and redundancy by synergistically targeting the same transcript $[15,34]$. The latter scenario may be the case in our study where the overexpression of miR-23a, -23b and -28 caused the endogenous downregulation of the other miRNAs that suggests a level of redundancy and cooperativity in their regulation and function. Furthermore, the combined overexpression of 
A

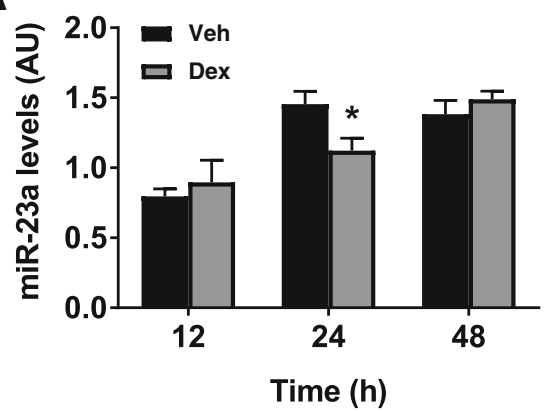

C

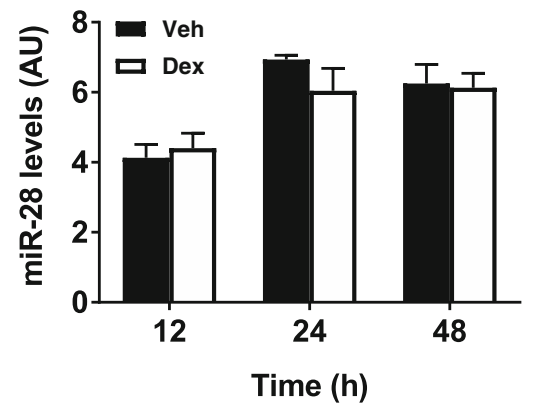

B

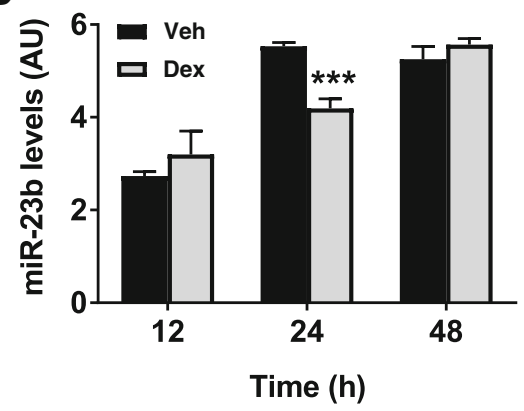

D

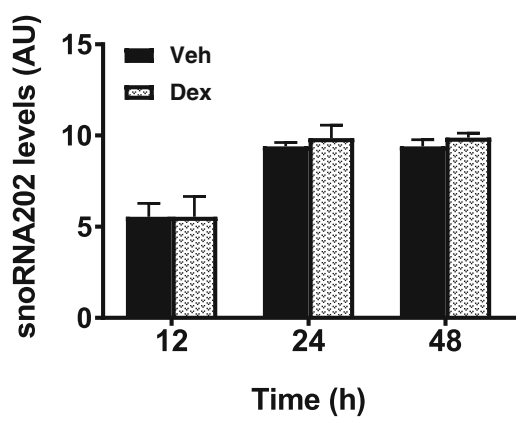

Fig. 4 Endogenous miRNA levels following dexamethasone treatment. a Endogenous miR-23a, (b) miR-23b, (c) miR-28, and (d) snoRNA202 levels over time $(\mathrm{h})$ following $10 \mu \mathrm{M}$ dexamethasone (Dex) treatment. Data are representative of two independent experiments with $\mathrm{n}=4$ per sample group; ${ }^{*} p<0.05$ and ${ }^{* * *} p<0.001$ to DMSO vehicle (Veh). Expression levels are presented as arbitrary units (AU)

the three miRNAs in the presence of dexamethasone prevented the increase in NDRG2 translation. This outcome maybe a reflection of the modulatory regulation of NDRG2 in stress scenarios but also emphasises the roles miRNAs play in disease and stress conditions experienced in skeletal muscle tissue. For example, miR-206-deficient mice display normal neuromuscular junction (NMJ) formation in healthy skeletal muscle although following the introduction of stress such as an acute injury causing muscle regeneration, new NMJ formation was impaired [9]. Furthermore, miR-206's deficiency exacerbated disease progression in a mouse model of motor neurone disease [9]. Whether all three miRNAs are required to act cooperatively or can function independently to inhibit NDRG2 increase during dexamethasone and other catabolic stress are yet unknown. However, since the effect of dexamethasone on NDRG2 levels is moderate, it is unlikely that individually, miR-23a, -23b and -28 will have a substantial effect on NDRG2 regulation. The potential co-regulation of NDRG2 by multiple miRNAs in skeletal muscle is further supported by a similar scenario reported recently in the lumbar region of embryonic sheep where target genes, including $N D R G 2$, are believed co-regulated by multiple miRNAs [38].

Each miRNA predicted to inhibit $N d r g 2$ in this study plays roles in skeletal muscle biological processes.
miR-23a, $-23 b$ and -28 are all linked to myogenesis and differentiation [11, 39-41] with miR-23a also associated with muscle wasting $[36,42,43]$. Previously, miR-23a was reported to decrease with dexamethasone in $\mathrm{C} 2 \mathrm{C} 12$ myotubes at $48 \mathrm{~h}$ post treatment [36], and in miR-23a transgenic mice, miR-23a reduced dexamethasone-induced muscle wasting potentially through the translational inhibition of the ubiquitin proteasomal E3 ligases, MAFbx/ atrogin-1 and MuRF1 [12]. Currently, these two studies do not delineate a clear role for miR-23a in dexamethasone induced-stress as its downregulation is counter-intuitive to a function involving MAFbx and MuRF1 inhibition. Here, we observed the downregulation of both miR-23a and miR-23b by dexamethasone, albeit at $24 \mathrm{~h}$, not $48 \mathrm{~h}$ post treatment. Previous studies have demonstrated that dexamethasone treatment increases $\mathrm{Ndrg} 2$ transcription in murine skeletal muscle cells [24] and in rat astrocytes [35, 44] with the induction of $N d r g 2$ by dexamethasone occurring indirectly through nuclear factor- $\mathrm{kB}$ or paired box gene 5 transcription factor binding [35]. Given our findings here, it is tempting to speculate that the decrease in miR-23a and -23b endogenous levels by dexamethasone may counteract their ability to block NDRG2's stress response increase to dexamethasone. Regardless, these findings suggest a novel regulatory mechanism of NDRG2 translation by miRNAs during stress conditions. Furthermore, it appears that these 

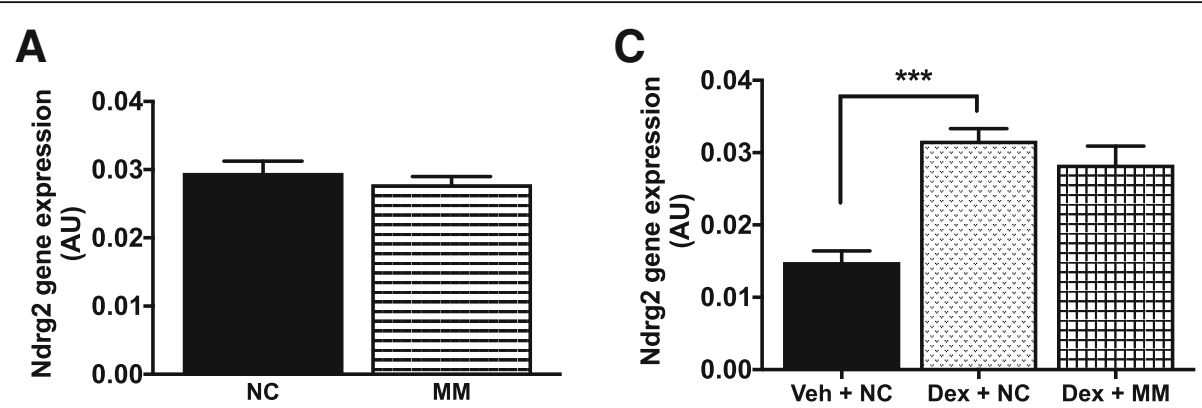

\section{B}

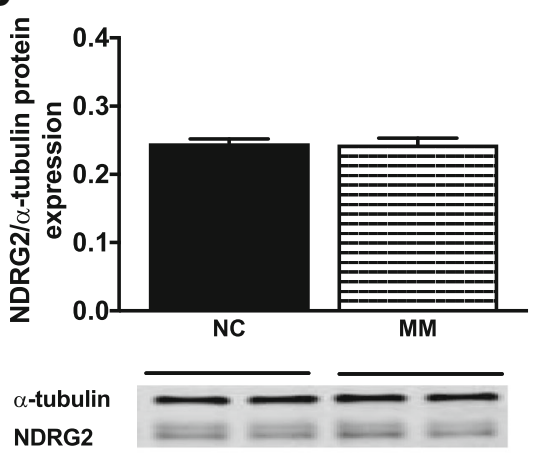

D

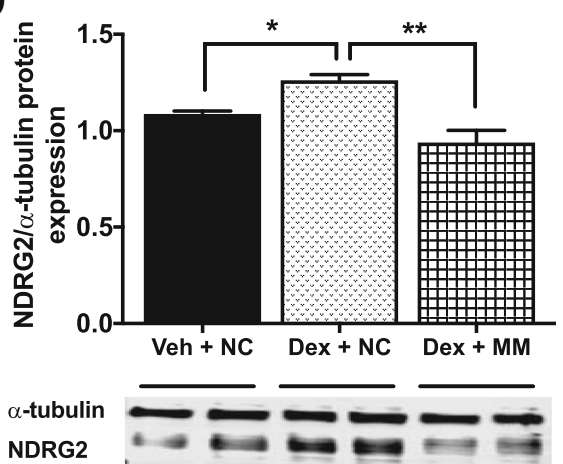

Fig. 5 The effect of combined miRNA overexpression on endogenous NDRG2 mRNA and protein levels under basal and catabolic conditions. miR-23a, -23b and -28 were co-transfected into myotubes and their combined effects on NDRG2 regulation was measured in the presence or absence of $48 \mathrm{~h}$ treatment with $10 \mu \mathrm{M}$ dexamethasone. a Ndrg2 mRNA and (b) NDRG2 protein levels under basal conditions following miRNA cotreatment (striped bar) or negative control miRNA (NC, black bar) transfection. c Ndrg2 mRNA and (d) NDRG2 protein levels following the combined effects of DMSO vehicle and negative control miRNA treatment (Veh + NC; grey bar), dexamethasone and negative control miRNA treatment (Dex + NC; stippled bar), or dexamethasone and miRNA mix treatment (Dex + MM; cross-hatched bar). Data are representative of two independent experiments with $n=4$ per sample group, and expression levels are presented as arbitrary units (AU). ${ }^{*} p<0.05,{ }^{* *} p<0.01$ or ${ }^{* * *} p<$ 0.001 to Dex + NC treatment

miRNAs function through their binding to the 3'UTR of $N d r g 2$, rather than degradation of $N d r g 2$ transcripts, two known mechanisms by which miRNAs can function to control gene and protein expression (reviewed in [45]).

While miR-23a and -23b have not been described elsewhere to target human or rodent $N d r g 2$, miR-28-5p was reported recently to target and suppress human NDRG2 in chronic lymphocytic leukemia cells [46]. This miRNA regulation was proposed as a mechanism to help suppress NDRG2 expression in cancer cells along with epigenetic silencing. This study used in silico and similar in vitro validation approaches involving dual luciferase assays in HEK293T where the authors identified miR-28 to bind to a predicted target sequence in the 3'UTR region of human NDRG2 [46], which helps validate our findings reported here of mouse $N d r g 2$ regulation by miR-28-5p.

\section{Conclusion}

Here, novel co-regulation of mouse $N d r g 2$ by miRNAs under specific stress conditions in skeletal muscle cells is described. However, the in vitro overexpression of the miRNAs in the presence of dexamethasone represents non-physiological conditions for the regulation of $\mathrm{Ndrg} 2$.
Whether miR-23a, $-23 \mathrm{~b}$ and -28 act cooperatively under similar stress conditions in vivo, and whether Ndrg2 represents a novel therapeutic target in catabolic muscle wasting conditions remains to be determined.

\section{Additional files}

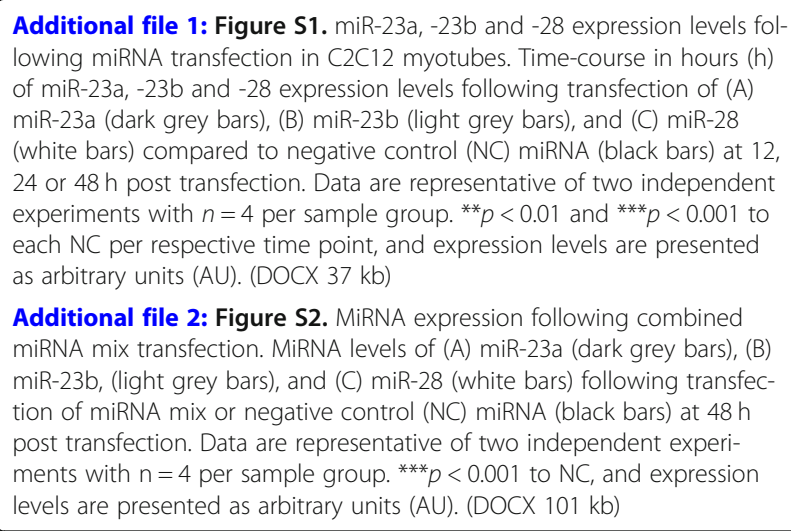

Abbreviations

3'UTR: 3' untranslated region; AU: arbitrary units; miRNA: microRNA;

NC: negative control; NDRG2: N-myc downstream-regulated gene 2 


\section{Acknowledgements}

Not applicable.

\section{Funding}

This research did not receive any specific grant from funding agencies in the public, commercial, or not-for-profit sectors. Seed funding for this study was received from IPAN, Deakin University.

\section{Availability of data and materials}

All data generated or analysed during this study are included in this article.

\section{Authors' contributions}

BAM performed in silico miRNA analyses, cell culture experiments, gene and protein expression measurements, statistical analyses and was a major contributor in writing the manuscript. RI contributed to cell culture experiments, protein expression measurements, and edited the manuscript. MK produced all luciferase plasmid constructs and edited the manuscript. AR assisted with conceptualisation of the study, supervision of BAM and edited the manuscript. VF conceptualised the study, performed the luciferase assays, wrote the majority of the manuscript and supervised BAM. All authors have read and approved the final manuscript.

\section{Ethics approval and consent to participate} Not applicable.

\section{Consent for publication}

Not applicable.

\section{Competing interests}

The authors declare that they have no competing interests.

\section{Publisher's Note}

Springer Nature remains neutral with regard to jurisdictional claims in published maps and institutional affiliations.

\section{Received: 1 May 2019 Accepted: 13 May 2019}

\section{Published online: 28 May 2019}

\section{References}

1. Chen JF, Mandel EM, Thomson JM, Wu Q, Callis TE, Hammond SM, et al. The role of microRNA-1 and microRNA-133 in skeletal muscle proliferation and differentiation. Nat Genet. 2006;38(2):228-33.

2. Kim HK, Lee YS, Sivaprasad U, Malhotra A, Dutta A. Muscle-specific microRNA miR-206 promotes muscle differentiation. J Cell Biol. 2006;174(5): 677-87.

3. Naguibneva I, Ameyar-Zazoua M, Polesskaya A, Ait-Si-Ali S, Groisman R, Souidi M, et al. The microRNA miR-181 targets the homeobox protein HoxA11 during mammalian myoblast differentiation. Nat Cell Biol. 2006;8(3): 278-84.

4. Coffey VG, Hawley JA. The molecular bases of training adaptation. Sports Med. 2007;37(9):737-63.

5. Hitachi K, Tsuchida K. Role of microRNAs in skeletal muscle hypertrophy. Front Physiol. 2013:4:408

6. McCarthy JJ, Esser KA. MicroRNA-1 and microRNA-133a expression are decreased during skeletal muscle hypertrophy. J Appl Physiol (1985). 2007; 102(1):306-13.

7. Anderson C, Catoe H, Werner R. MIR-206 regulates connexin43 expression during skeletal muscle development. Nucleic Acids Res. 2006:34(20):5863-71.

8. van Rooij E, Quiat D, Johnson BA, Sutherland $L B$, Qi X, Richardson JA, et al. A family of microRNAs encoded by myosin genes governs myosin expression and muscle performance. Dev Cell. 2009;17(5):662-73.

9. Williams AH, Valdez G, Moresi V, Qi X, McAnally J, Elliott JL, et al. MicroRNA206 delays ALS progression and promotes regeneration of neuromuscular synapses in mice. Science. 2009;326(5959):1549-54

10. Wang B, Zhang C, Zhang A, Cai H, Price SR, Wang XH. MicroRNA-23a and MicroRNA-27a mimic exercise by ameliorating CKD-induced muscle atrophy. J Am Soc Nephrol. 2017:28(9):2631-40.

11. Wang L, Chen X, Zheng Y, Li F, Lu Z, Chen C, et al. MiR-23a inhibits myogenic differentiation through down regulation of fast myosin heavy chain isoforms. Exp Cell Res. 2012;318(18):2324-34.
12. Wada S, Kato Y, Okutsu M, Miyaki S, Suzuki K, Yan Z, et al. Translational suppression of atrophic regulators by microRNA-23a integrates resistance to skeletal muscle atrophy. JBC. 2011;286(44):38456-65.

13. McCarthy JJ, Esser KA, Peterson CA, Dupont-Versteegden EE. Evidence of MyomiR network regulation of beta-myosin heavy chain gene expression during skeletal muscle atrophy. Physiol Genomics. 2009;39(3):219-26.

14. Wang XH. MicroRNA in myogenesis and muscle atrophy. Curr Op Clin Nut Met Care. 2013;16(3):258-66.

15. Liu N, Bassel-Duby R. Regulation of skeletal muscle development and disease by microRNAs. Results Probl Cell Differ. 2015;56:165-90.

16. Melotte V, Qu X, Ongenaert M, van Criekinge W, de Bruine AP, Baldwin HS, et al. The N-myc downstream regulated gene (NDRG) family: diverse functions, multiple applications. FASEB J. 2010;24(11):4153-66.

17. Feng $L$, Xie $Y$, Zhang $H$, Wu $Y$. Down-regulation of NDRG2 gene expression in human colorectal cancer involves promoter methylation and microRNA650. BBRC. 2011:406(4):534-8

18. Lango-Chavarria M, Chimal-Ramirez GK, Ruiz-Tachiquin ME, EspinozaSanchez NA, Suarez-Arriaga MC, Fuentes-Panana EM. A 22q11.2 amplification in the region encoding microRNA-650 correlates with the epithelial to mesenchymal transition in breast cancer primary cultures of Mexican patients. Internat J Oncol. 2017:50(2):432-40.

19. Guo YJ, Liu JX, Guan YW. Hypoxia induced upregulation of miR-301a/b contributes to increased cell autophagy and viability of prostate cancer cells by targeting NDRG2. Europ Rev Med Pharmacol Sci. 2016;20(1):101-8.

20. Wang W, Liu M, Guan Y, Wu Q. Hypoxia-responsive mir-301a and mir-301b promote radioresistance of prostate cancer cells via downregulating NDRG2. Med Sci Monit. 2016:22:2126-32.

21. Wang J, Xie C, Pan S, Liang Y, Han J, Lan Y, et al. N-myc downstreamregulated gene 2 inhibits human cholangiocarcinoma progression and is regulated by leukemia inhibitory factor/MicroRNA-181c negative feedback pathway. Hepatology. 2016;64(5):1606-22.

22. Agosta C, Laugier J, Guyon L, Denis J, Bertherat J, Libe R, et al. MiR-483-5p and miR-139-5p promote aggressiveness by targeting $\mathrm{N}$-myc downstreamregulated gene family members in adrenocortical cancer. Int J Cancer. 2018; 143(4):944-57.

23. Zhou RH, Kokame K, Tsukamoto Y, Yutani C, Kato H, Miyata T. Characterization of the human NDRG gene family: a newly identified member, NDRG4, is specifically expressed in brain and heart. Genomics. 2001;73(1):86-97.

24. Foletta VC, Prior MJ, Stupka N, Carey K, Segal DH, Jones S, et al. NDRG2, a novel regulator of myoblast proliferation, is regulated by anabolic and catabolic factors. J Physiol. 2009;587(Pt 7:1619-34.

25. Hu XL, Liu XP, Deng YC, Lin SX, Wu L, Zhang J, et al. Expression analysis of the NDRG2 gene in mouse embryonic and adult tissues. Cell Tiss Res. 2006;325(1):67-76.

26. Anderson KJ, Russell AP, Foletta VC. NDRG2 promotes myoblast proliferation and caspase 3/7 activities during differentiation, and attenuates hydrogen peroxide, but not palmitate-induced toxicity. FEBS Open bio. 2015:5:668-81.

27. Foletta VC, Brown EL, Cho Y, Snow RJ, Kralli A, Russell AP. Ndrg2 is a PGC1 alpha/ERRalpha target gene that controls protein synthesis and expression of contractile-type genes in C2C12 myotubes. Biochim Biophys Acta. 2013; 1833(12):3112-23.

28. Betel D, Koppal A, Agius P, Sander C, Leslie C. Comprehensive modeling of microRNA targets predicts functional non-conserved and non-canonical sites. Genome Biol. 2010;11(8):R90.

29. Betel D, Wilson M, Gabow A, Marks DS, Sander C. The microRNA.org resource: targets and expression. Nucleic Acids Res. 2008;36:D149-D53.

30. Dweep H, Gretz N. miRWalk2.0: a comprehensive atlas of microRNA-target interactions. Nat Methods. 2015;12(8):697.

31. Soriano-Arroquia A, House L, Tregilgas L, Canty-Laird E, Goljanek-Whysall K. The functional consequences of age-related changes in microRNA expression in skeletal muscle. Biogerontology. 2016;17:641-54.

32. Alvarez-Saavedra $E_{1}$ Horvitz HR. Many families of $C$. elegans microRNAs are not essential for development or viability. Curr Biol. 2010;20(4):367-73

33. Brenner JL, Jasiewicz KL, Fahley AF, Kemp BJ, Abbott AL. Loss of individual microRNAs causes mutant phenotypes in sensitized genetic backgrounds in C. elegans. Curr Biol. 2010;20(14):1321-5.

34. Small EM, Olson EN. Pervasive roles of microRNAs in cardiovascular biology. Nature. 2011:469(7330):336-42.

35. Takahashi K, Saitoh A, Yamada M, Iwai T, Inagaki M, Yamada M Dexamethasone indirectly induces Ndrg2 expression in rat astrocytes. J Neurosci Res. 2012;90(1):160-6. 
36. Hudson MB, Woodworth-Hobbs ME, Zheng B, Rahnert JA, Blount MA, Gooch $J L$, et al. miR-23a is decreased during muscle atrophy by a mechanism that includes calcineurin signaling and exosome-mediated export. AJP - Cell Physiol. 2014;306(6):C551-C8.

37. O'Rourke JR, Georges SA, Seay HR, Tapscott SJ, McManus MT, Goldhamer DJ, et al. Essential role for Dicer during skeletal muscle development. Dev Biol. 2007;311(2):359-68.

38. Zhang X, Li C, Li X, Liu Z, Ni W, Hazi W, et al. Expression profiles of MicroRNAs from multiple lumbar spine in sheep. Gene. 2018;678:105-14.

39. Dmitriev P, Barat A, Polesskaya A, O'Connell MJ, Robert T, Dessen P, et al. Simultaneous miRNA and mRNA transcriptome profiling of human myoblasts reveals a novel set of myogenic differentiation-associated miRNAs and their target genes. BMC Genomics. 2013;14(1):265-84.

40. Lozano-Velasco E, Vallejo D, Esteban FJ, Doherty C, Hernandez-Torres F, Franco D, et al. A Pitx2-MicroRNA pathway modulates cell proliferation in myoblasts and skeletal-muscle satellite cells and promotes their commitment to a myogenic cell fate. Mol Cell Biol. 2015;35(17):2892-909.

41. Mercatelli N, Fittipaldi S, De Paola E, Dimauro I, Paronetto MP, Jackson MJ, et al. MiR-23-TrxR1 as a novel molecular axis in skeletal muscle differentiation. Sci Rep. 2017;7(1):7219.

42. Li G, Li QS, Li WB, Wei J, Chang WK, Chen Z, et al. miRNA targeted signaling pathway in the early stage of denervated fast and slow muscle atrophy. Neural Regen Res. 2016;11(8):1293-303.

43. Zhang A, Li M, Wang B, Klein JD, Price SR, Wang XH. miRNA-23a/27 attenuates muscle atrophy and renal fibrosis through muscle-kidney crosstalk. J Cachexia Sarcopenia Muscle. 2018;9(4):755-70.

44. Nichols NR. Ndrg2, a novel gene regulated by adrenal steroids and antidepressants, is highly expressed in astrocytes. Ann N Y Acad Sci. 2003; 1007:349-56.

45. Zacharewicz E, Lamon S, Russell AP. MicroRNAs in skeletal muscle and their regulation with exercise, ageing, and disease. Front Physiol. 2013;266:1-11.

46. Yang YQ, Tian T, Zhu HY, Liang JH, Wu W, Wu JZ, et al. NDRG2 mRNA levels and miR-28-5p and miR-650 activity in chronic lymphocytic leukemia. BMC Cancer. 2018;18(1):1009-18.

Ready to submit your research? Choose BMC and benefit from:

- fast, convenient online submission

- thorough peer review by experienced researchers in your field

- rapid publication on acceptance

- support for research data, including large and complex data types

- gold Open Access which fosters wider collaboration and increased citations

- maximum visibility for your research: over $100 \mathrm{M}$ website views per year

At $\mathrm{BMC}$, research is always in progress.

Learn more biomedcentral.com/submissions 